\title{
New Efficient Timing and Frequency Error Estimation in OFDM
}

\author{
A. P. Rathkanthiwar ${ }^{1}$ and A. S. Gandhi ${ }^{2}$ \\ ${ }^{1}$ Department of Electronics Engineering, Priyadarshini College of Engineering, \\ Nagpur, MS, India, anagharathkanthiwar@yahoo.co.in \\ ${ }^{2}$ Professor, Department of Electronics, Vesvesvarya National Institute of \\ Technology, Nagpur, MS, India, abhay4083@yahoo.co.in
}

Received 24 August 2013; Accepted 27 November 2013;

Publication 23 January 2014

\begin{abstract}
Orthogonal frequency division multiplexing (OFDM) technique is being applied extensively to high data rate digital transmission as it is a bandwidth efficient modulation scheme. In OFDM system, intersymbol interference (ISI) and intercarrier interference (ICI) occur due to synchronization errors. Fast, simple and robust synchronization algorithms are necessary for OFDM systems. In OFDM downlink transmission, every terminal perform synchronization by exploiting reference symbols called training symbols of received frame. In this paper we are presenting very simple method of synchronization which uses training symbol to determine timing and frequency synchronization error. Proposed synchronization techniques result in better performance with respect to all the parameters despite being simple.
\end{abstract}

Keywords: Synchronization, orthogonal frequency division multiplexing (OFDM), timing estimation, CFO, CFO estimation.

\section{Introduction}

Orthogonal frequency division multiplexing (OFDM) is a bandwidth efficient modulation scheme for high speed data communication in frequency-selective multi-path fading channels [1]. The mitigation of frequency selectivity in multi-path fading channels is possible with OFDM, because the frequency

Journal of Cyber Security, Vol. 2 No. 3 \& 4 , 291-306.

doi: 10.13052/jcsm2245-1439.235

(c) 2014 River Publishers. All rights reserved. 
selective fading channel is transformed into multiple flat fading channels, one for each sub-carrier. The orthogonality among sub-carriers makes a more compact signal bandwidth for a given data rate. Furthermore, it provides larger flexibility by allowing independent selection of the modulation parameters (like the constellation size and coding scheme) over each sub-carrier. OFDM Modulation can be realized with Inverse Fast Fourier Transform (IFFT). Due to all these favorable features, many digital transmission systems have adopted OFDM as the modulation technique such as digital video broadcasting terrestrial TV (DVB-T), digital audio broadcasting (DAB), terrestrial integrated services digital broadcasting (ISDB-T), digital subscriber line (xDSL) etc. Now it is being used in packet based systems like multimedia mobile access communications (MMAC), and the fixed wireless access (FWA) system in IEEE 802.16.3 standard [2]. It has become fundamental technology in the future 3GPP LTE and 4G-multimedia mobile communication systems.

However, the OFDM transmission is sensitive to receiver synchronization imperfections. The symbol timing synchronization error cause intersymbol interference (ISI) and the frequency synchronization error is one of the reasons for intercarrier interference (ICI). In OFDM, frequency synchronization errors are actually Carrier Frequency Offsets (CFO) and are generally caused by unmatched local oscillators at the two ends of the communication links, Doppler shifts or phase noise introduced by nonlinear channel[3]. Thus, synchronization is a crucial issue in an OFDM receiver design. Task of timing synchronization of an orthogonal frequency division multiplexing (OFDM) receiver, requires alignment of the discrete Fourier transform (DFT) segments with OFDM symbol boundaries [4]. Timing alignment errors may occur in cases where the DFT aperture contains part of the guard interval that has been distorted by intersymbol interference (ISI). Synchronization techniques for OFDM systems can be classified as either blind or data-aided. Blind approaches exploit the inherent redundancy in the OFDM signal structure for example, cyclic prefix $(\mathrm{CP})$. Even though blind techniques have the advantage of not requiring extra overhead, their performance usually degrades when the noise level is high or the channel distortion is severe. Data-aided techniques offer the advantage of superior performance in low SNR applications at the expense of reduced spectral efficiency[4]. These techniques make use of reference symbols embedded into the transmitted signal. One way of embedding a reference symbol into a transmitted signal is to prefix it to the beginning of the time-domain waveform in the form of a preamble. Reliable synchronization is one of the key factors determining the transmission performance in 
communication channels. Various schemes for time and frequency estimation for imperfect communication links have been explained in the literature.

\section{System Model}

In OFDM systems, the data is modulated in blocks by means of a Fast Fourier transform (FFT). Data stream is mapped into $\mathrm{N}$ complex symbols in frequency domain. These $\mathrm{N}$ complex symbols are modulated on $\mathrm{N}$ subcarriers by using $\mathrm{N}$-point inverse fast Fourier transform (IFFT) and the time domain samples are computed using the well-known IFFT formula

$$
x(n)=\frac{1}{\sqrt{N}} \sum_{k=0}^{N-1} d_{k}(n) e^{\frac{j 2 \pi k n}{N}} \quad n=0,1, \ldots N-1
$$

Received signal can be modeled as

$$
r(n)=x(n-\theta) e^{j 2 \pi n \epsilon}+w(n)
$$

Here $\theta$ represents the unknown integer-valued time offset, $\varepsilon$ is the carrier frequency offset (CFO), $\mathrm{w}(\mathrm{n})$ is complex additive white Gaussian noise and $\mathrm{x}(\mathrm{n})$ is transmitted signal.

\section{Timing Error Estimation}

Timing error or timing offset $\theta$ occurs because of channel delay and multipath dispersion. Due to this timing error the receiver's time-domain FFT window spans samples from two consecutive OFDM symbols. This results in inter-OFDM symbol interference leading to BER degradation [6]. The requirements on the time offset estimator are determined by the difference in length between the cyclic prefix (CP) and the channel impulse response (CIR) [5]. This difference is the part of the cyclic prefix that is not affected by the previous symbol due to the channel dispersion, as shown in Figure 1. As long as a symbol time offset estimate does not exceed this difference, the orthogonality of the subcarriers is preserved, and a time offset within this interval only results in a phase rotation of the subcarrier constellations. The closer the time offset estimate is to the true offset, the shorter the cyclic prefix needs to be, reducing the overhead in the system. 


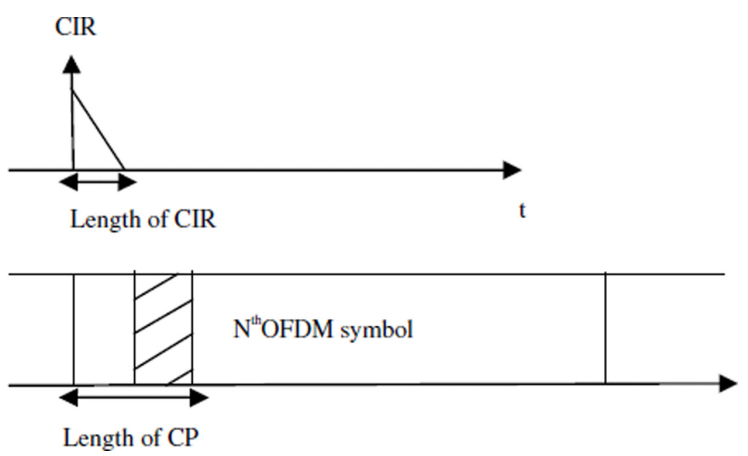

Figure 1 The time offset requirements

\section{Effect of Timing Error}

The effect of uncompensated timing error on system performance can be described by figure 2 . As shown in figure 2 the tail of each received block extends over the first L - 1 samples of the successive block as a consequence of multipath dispersion. If cyclic prefix is greater than CIR duration ( 0 to L-1) then there is certain interval which is not affected by previous block. As long as the DFT window starts anywhere in this interval, no Inter Symbol Interference (ISI) is present at the FFT output. This situation occurs whenever the timing error $\theta$ belongs to interval $-\mathrm{Ng}+\mathrm{L}-1 \leq \theta \leq 0$ and only results in a cyclic shift of the received OFDM block [7]. Thus, recalling the time-shift property of the Fourier transform and assuming perfect frequency synchronization the DFT output over the $\mathrm{n}^{\text {th }}$ subcarrier takes the form

$$
R(k)=e^{\frac{j 2 \pi k \theta}{N}} H(k) d(k)+w(k)
$$

Here

$$
H(k)=\sum_{l=0}^{L-1} h(l) e^{-j 2 \pi k l} / N
$$

Where $h(l)$ is complex channel impulse response, $\mathrm{d}$ corresponds to OFDM symbol.

The equation of $\mathrm{R}(\mathrm{k})$ indicates that timing error $\theta$ appears as a linear phase across subcarriers. On the other hand, if the timing error is outside the interval $-\mathrm{Ng}+\mathrm{L}-1 \leq \theta \leq 0$, samples at the FFT input will be contributed by two adjacent OFDM blocks causing Inter Symbol Interference (ISI). In addition to ISI, this results in a loss of orthogonality among subcarriers which in turn, 


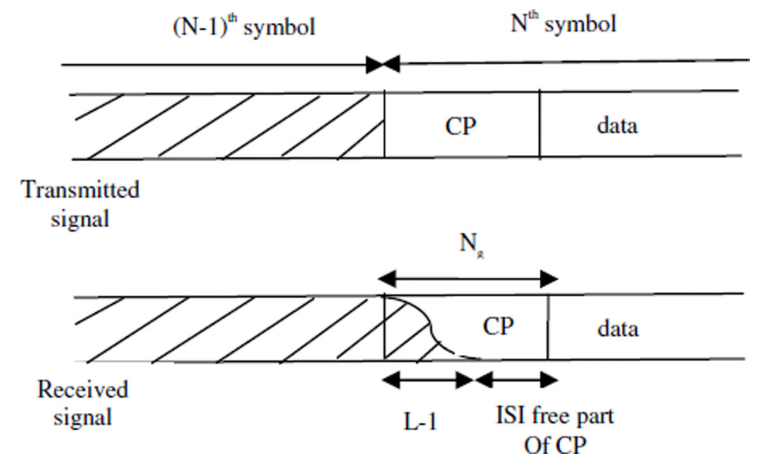

Figure 2 Partial overlapping between received blocks due to multipath dispersion

generates Inter Carrier Interference (ICI). In this case, the $\mathrm{k}^{\text {th }}$ DFT output is given by

$$
R(k)=e^{\frac{j 2 \pi k \theta}{N}} \alpha(\theta) H(k) d(k)+I(k, \theta)+w(k)
$$

Where I $(\mathrm{k}, \theta)$ is the introduced ISI. Due to the introduced ISI and the phase rotation, there is slight magnitude attenuation $\alpha(\theta)$ in the signal.

\section{Timing Error Estimation}

In the process of synchronization in OFDM systems, errors or offsets are estimated and then corrected using the estimated values. In order to perform the FFT demodulation correctly, the symbol timing synchronization must be done to determine the starting point (i.e. FFT window) of the OFDM symbol. It is important to solve symbol timing synchronization problem first during the design process of an OFDM receiver. The conventional algorithms for the symbol timing estimation in time domain are MLE (Maximum Likelihood Estimation) utilizing the cyclic prefix of the OFDM symbols. The most representative algorithm was proposed by J. J. Van de Beek [2]. However, good performance achieved only under the AWGN channel. When the channel condition becomes severely degraded, data is badly contaminated by ISI. T. M. Schmidl and Cox (S and C) introduced a new method making use of the training symbol called reference block which is placed in front of data symbols in the frame [7]. Training symbols or reference blocks has repetitive structure in the time domain and are exploited for error estimation purpose at the receiver. In this case, timing estimator can be designed by searching for the peak of the correlation among repetitive parts called as 
timing metric. Training symbol used by Schmidt and Cox (S \& C) composed of two identical halves of length N/2. It has better performance compared to that proposed by J. J. Van de Beek. Unfortunately, timing metric of this algorithm exhibits a large plateau that may greatly reduce the estimation accuracy[6]. To reduce uncertainty due to timing metric plateau, Shi and Serpedin $(S \& S)$ used a training block composed of four repetitive parts $[+\mathrm{B}+\mathrm{B}-\mathrm{B}+\mathrm{B}]$ with a sign inversion in the third segment [8]. Here $B$ is PN sequence of length N/4. More accurate timing estimate is obtained. Jung Ju Kim, Jungho Noh, and Kyung Hi Chang presented a scheme in their paper [9] which uses a training symbol composed of four parts [B $\mathrm{B}^{*} \mathrm{~B} \mathrm{~B}^{*}$ ]. Here * represents conjugate of quantity. This method reduces plateau and it can achieve more accurate timing offset estimation. Since CFO is usually unknown at this stage, it is desirable that the timing recovery scheme be robust against possibly large frequency offsets. The first step of the timing estimation is the detection of a new frame in the received data stream. For this purpose, timing metric is continuously monitored and the start of a frame is declared whenever it overcomes a given threshold or when a peak is observed in the timing metric

\section{Proposed Timing Error Estimator}

The training symbol used for proposed algorithm is similar to the first training symbol used in [7] and has two identical halves each of length N/2 in time domain. This training symbol is used as a preamble. The Timothy M. Schmidl and Donald C. Cox (S \& C) method [7] uses autocorrelation function (ACF) to compute timing metric. The formula is

$$
(P)_{A C F}(n)=\sum_{k=0}^{\frac{N}{2}-1} r(n+k+N / 2) r^{*}(n+k)
$$

Note that $\mathrm{n}$ is a time index corresponding to the first sample in a window of $\mathrm{N}$ samples. This window slides along in time as the receiver searches for the first training symbol. For this purpose, timing metric is continuously monitored and the start of a frame is declared whenever it overcomes a given threshold. Unfortunately the timing metric of the $\mathrm{S} \& \mathrm{C}$ algorithm exhibits a large plateau that may greatly reduce the estimation accuracy. This timing metric is shown in figure 3 (a). However cross correlation function (CCF) gives better timing metric. The corresponding equation is 


$$
P_{C C F}(n)=\sum_{k=0}^{N-1} r(n+k) s^{*}(k)
$$

Timing metric of this CCF function produces three peaks as shown in figure3 (b). Major peak corresponds to full symbol match and two minor peaks corresponds to half symbol match. Start of a frame is declared and timing error is estimated whenever maximum is detected. However minor peaks may cause trouble in severe noise conditions in the estimation process. Drawbacks of both ACF \& CCF timing metric is overcome by multiplying both functions over fixed interval. The resultant timing metric shows single and clear major peak as shown in figure 4. This results in improved estimation performance. Metric of this proposed algorithm is obtained by using

$$
P(n)=P_{A C F} * P_{C C F}
$$

Arrival of frame is declared whenever a peak is detected and timing error is estimated by

$$
\widehat{\theta}=\arg (\max (P(n)))
$$

Here timing metric shown in figures 3 and 4 are obtained in the presence of timing offset. Timing error is estimated using proposed algorithms and $\mathrm{S}$ and $\mathrm{C}$ algorithm. The parameters used for performance analysis of these proposed timing error estimators are mean error occurred in timing error estimation, standard deviation of timing error estimation, and probability of correct detection.

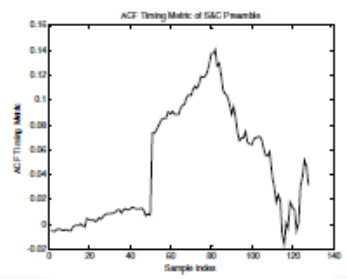

a Timing Metric of $\mathrm{ACF}$

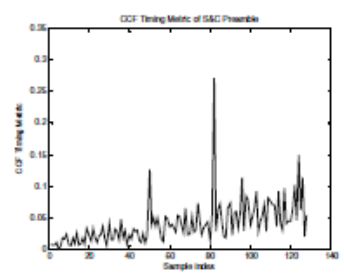

b Timing Metric of CCF

Figure 3 Timing Metric of ACF b Timing Metric of CCF 


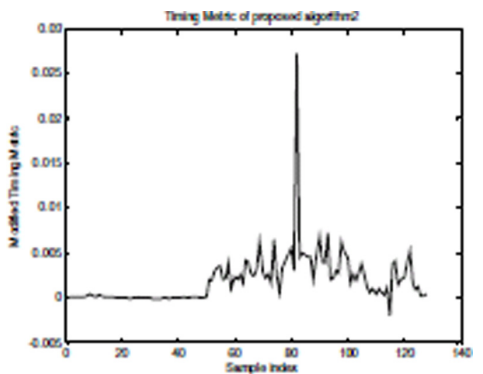

Figure 4 Timing Metric of proposed algorithm2

\section{Frequency Offset Estimation}

The FFT filter's frequency response extends over the whole frequency range and the very narrow spacing between sub-carriers all contribute to the sensitivity of OFDM systems to frequency synchronization errors[3]. Carrier frequency offset if present causes a phase rotation. This phase rotation effect is clearly seen through constellation diagram as shown in figure 5 .

These constellation diagram are obtained for 4QAM in the presence of $30 \mathrm{~dB}$ SNR noise and subcarrier spacing is $312.5 \mathrm{KHz}$.

Using the fact that phase separation between the two consecutive peaks of CCF function is proportional to CFO. Carrier frequency offset hence can be estimated using [10]

$$
\Delta f=\frac{\Delta \phi}{\pi T_{u} / 2}
$$

Here $\Delta \phi$ is phase difference between two peaks of CCF function which are separated by time duration of $\mathrm{T}_{\mathrm{u}} / 2$.
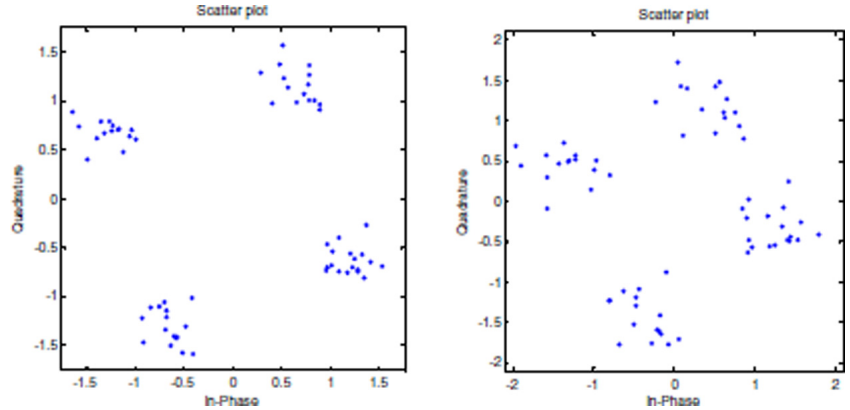

Figure 5 Phase rotation caused by $\mathrm{CFO}$ for $30 \mathrm{KHz}$ and $50 \mathrm{KHz}$ 


\section{Performance Evaluation}

\section{Simulation Parameters}

Performance of the proposed synchronization technique is evaluated by MATLAB $\AA^{1}{ }^{1}$ simulation over the AWGN and frequency-selective fading channels typical of broadband wireless communication systems. Specifically, as a fading channel model, this paper uses ITU channel model [11] for Vehicular Test Environment of type A and details of this channel model is given in table 1 below.

The other major simulation parameters are listed in table 2. Simulation is performed to obtain Mean error of timing estimation, Standard deviation of timing estimation, Detection probability, Bit error ratio etc. for performance measurement of proposed algorithm. For simulation in MATLAB, known fixed timing offset is applied to the OFDM frame[9] and the channel used is AWGN and Rayleigh channel as defined in table 2.

Table 1 Rayleigh channel details

\begin{tabular}{|l|l|l|}
\hline Tap & Channel A & \\
\hline & Relative Delay (ns) & Average Power (dB) \\
\hline 1 & 0 & 0.0 \\
2 & 310 & -1.0 \\
3 & 710 & -9.0 \\
4 & 1090 & -10.0 \\
5 & 1730 & -15.0 \\
6 & 2510 & -20.0 \\
\hline
\end{tabular}

Table 2 Simulation Parameters

\begin{tabular}{|l|l|}
\hline FFT length N & 64 \\
\hline Constellation mapping & 4 \& 16 QAM \\
\hline Subcarrier Spacing in KHz & 312.5 \\
\hline Useful OFDM Symbol Period & $3.2 \mathrm{e}-6$ \\
\hline Applied Timing Offset theta & 18 \\
\hline Number of simulation runs & 100 \\
\hline SNR range & 0 to $20 \mathrm{~dB}$ \\
\hline Number of Symbols & 30 \\
\hline
\end{tabular}

\footnotetext{
${ }^{1}$ MATLAB is a registered trademark of MathWorks Inc.
} 


\section{Symbol Timing Estimator Performance for AWGN Channel}

Timing offset estimation mean error obtained with proposed algorithms as a function of SNR values for 4 QAM and 16 QAM is shown in figure 6. For comparison $\mathrm{S}$ and $\mathrm{C}$ algorithm have also been considered. For both algorithms, timing mean error decreases with increase in SNR value. However proposed algorithm becomes perfect estimator for SNR values of $10 \mathrm{~dB}$ and above in case of 4QAM.

Similarly figure 7 shows standard deviation graph of timing error estimation for 4QAM and 16QAM obtained using these algorithms. Detection probability graph are shown in figure 8 . Detection probability increases with increase in SNR. Estimator corresponding to proposed algorithm achieves perfect synchronization for SNR value more than $10 \mathrm{~dB}$. For 16QAM performance improvement of proposed algorithm is more with respect to detection probability. The graphs shown in figures 6,7, 8 are for AWGN channel.
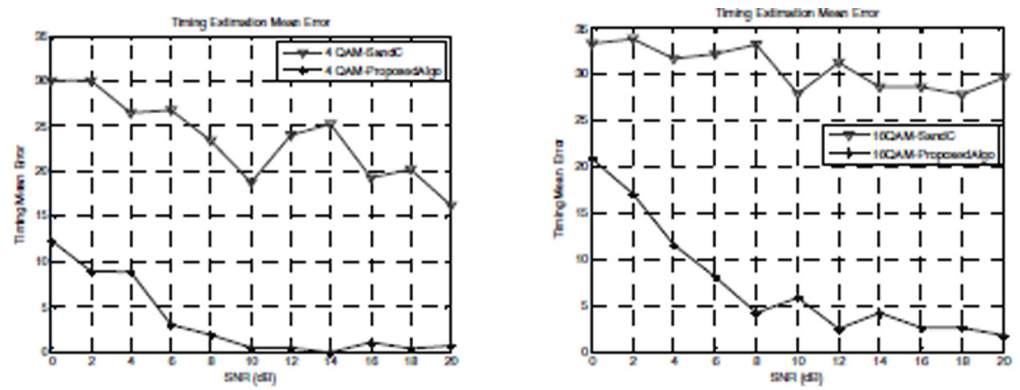

Figure 6 Timing Estimation Mean error for 4QAM and 16QAM
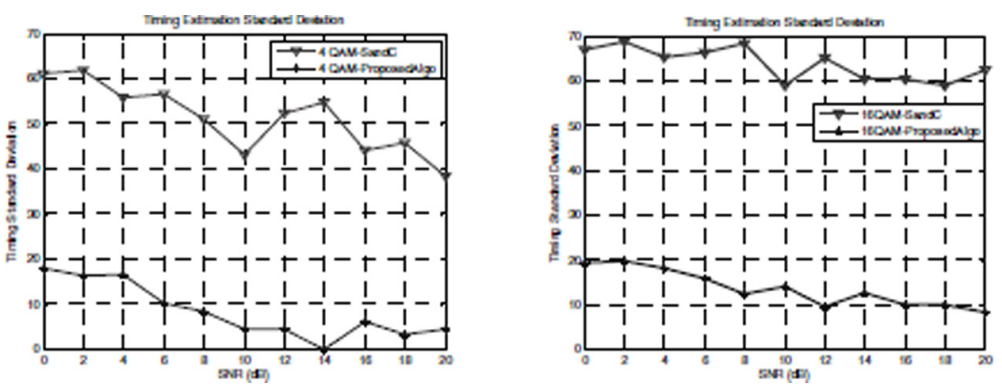

Figure 7 Timing Estimation Standard deviation for 4QAM and 16QAM 

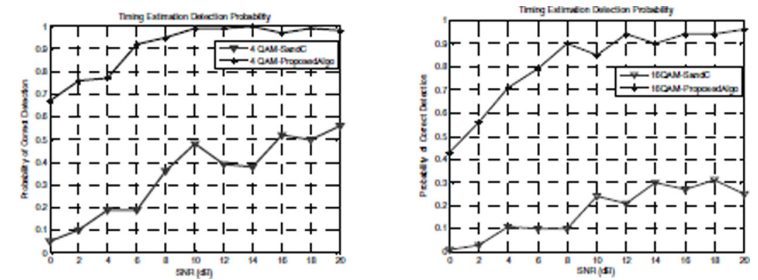

Figure 8 Detection Probability graph for 4QAM and 16QAM

\section{Symbol Timing Estimator Performance for Rayleigh Channel}

Results are also obtained for Rayleigh channel with parameters defined in table 1, using existing $\mathrm{S}$ and $\mathrm{C}$ algorithm and proposed algorithm. Resulting graphs for 4QAM and 16QAM are shown in figure 9, 10 respectively for mean error, standard deviation of timing error. From these figures it is noted that for Rayleigh environment also proposed algorithm give improved performance with respect to all parameters. Comparatively improvement in performance of proposed algorithm with respect to $\mathrm{S}$ and $\mathrm{C}$ algorithm is more in Rayleigh channel environment.
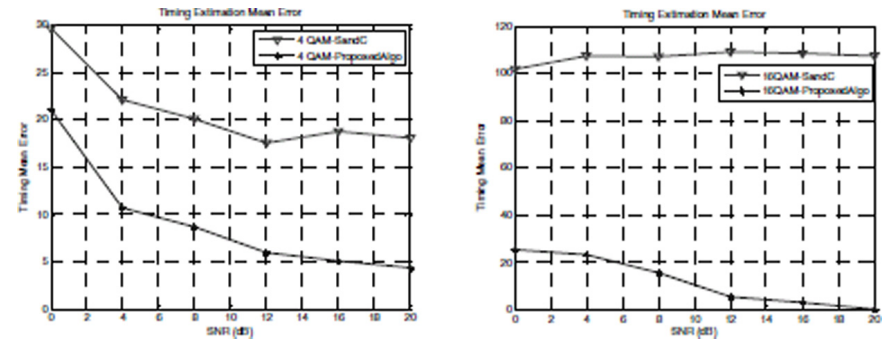

Figure 9 Timing Estimation Mean error for 4QAM and 16QAM
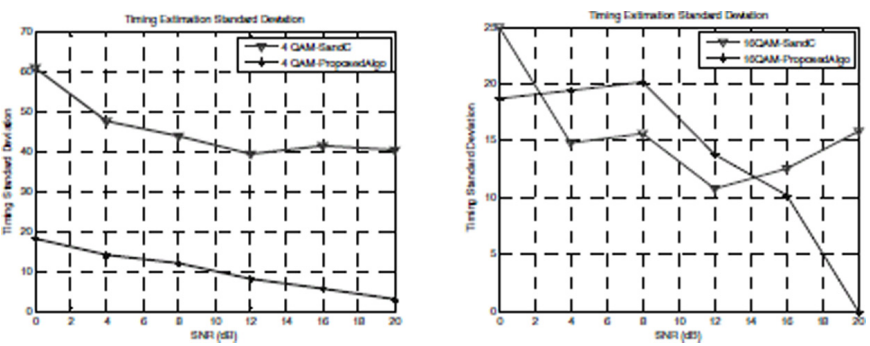

Figure 10 Timing Estimation Standard deviation for 4QAM and 16QAM 


\section{Overall BER Performance}

To check overall system performance using proposed timing offset estimator, a BER graph is obtained taking AWGN channel for 4QAM. At receiver timing error is estimated using algorithm. Estimated value is used to correct timing error and after FFT and demodulation BER values are obtained. BER graph obtained is shown in figure 10 .

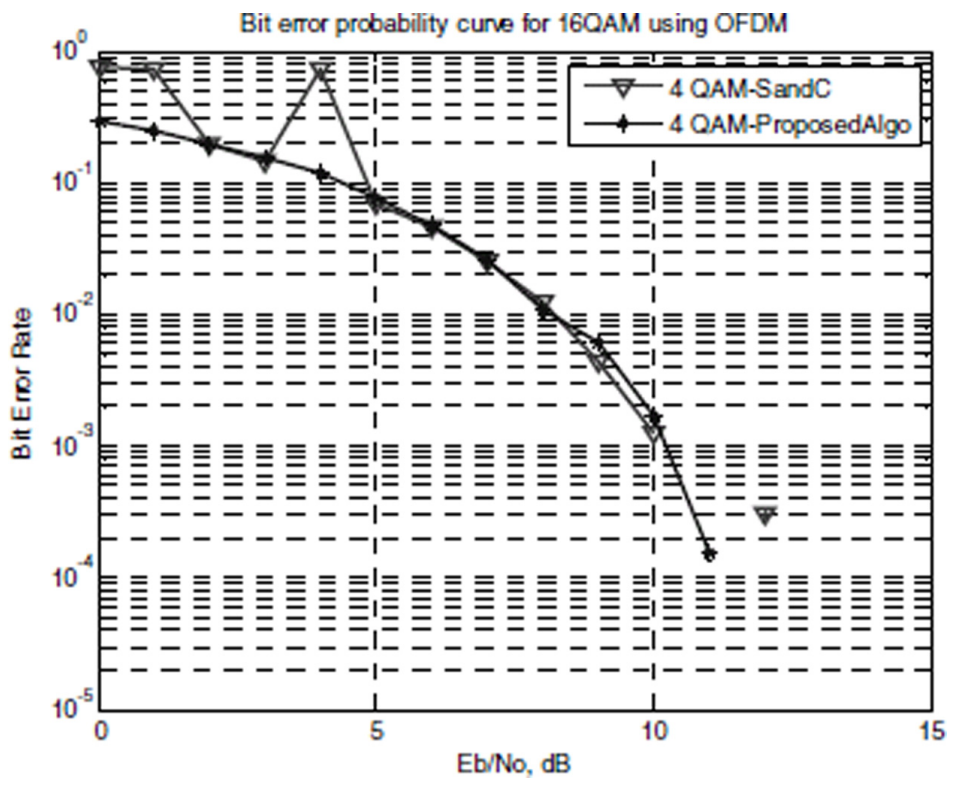

Figure 11 Bit Error Ratio graph for 4QAM, AWGN channel

As seen from figure 11, it is obvious that the proposed estimator significantly improves the BER performance compared to conventional S and C. It should be noted that, below $10 \mathrm{~dB}$ SNR, proposed estimators achieve more robustness than that of $\mathrm{S}$ and $\mathrm{C}$.

\section{Simulation for CFO Estimation}

Known value of CFO is inserted in the symbol carriers. Simulation for CFO estimation is then done by assuming perfect timing synchronization. Value of CFO is estimated using equation 11 by first calculating phase between peaks of CCF function in the presence of noise. Result shown in table 3 are obtained using parameters of table 2 and $20 \mathrm{~dB}$ SNR. Same CCF metric of timing synchronization is used here to estimate CFO. Figure 12 indicates the graph 
New Efficient Timing and Frequency Error Estimation in OFDM 303

Table 3 Actual and estimated value of CFO

\begin{tabular}{|l|l|}
\hline $\begin{array}{l}\text { Inserted value } \\
\text { of CFO in KHZ }\end{array}$ & $\begin{array}{l}\text { Estimated value } \\
\text { of CFO in KHZ }\end{array}$ \\
\hline 10 & 8.86 \\
\hline 20 & 17.26 \\
\hline 30 & 30.85 \\
\hline 40 & 41.01 \\
\hline 50 & 48.19 \\
\hline 60 & 58.97 \\
\hline 70 & 69.84 \\
\hline 80 & 80.5 \\
\hline 90 & 89.25 \\
\hline 100 & 100.58 \\
\hline
\end{tabular}

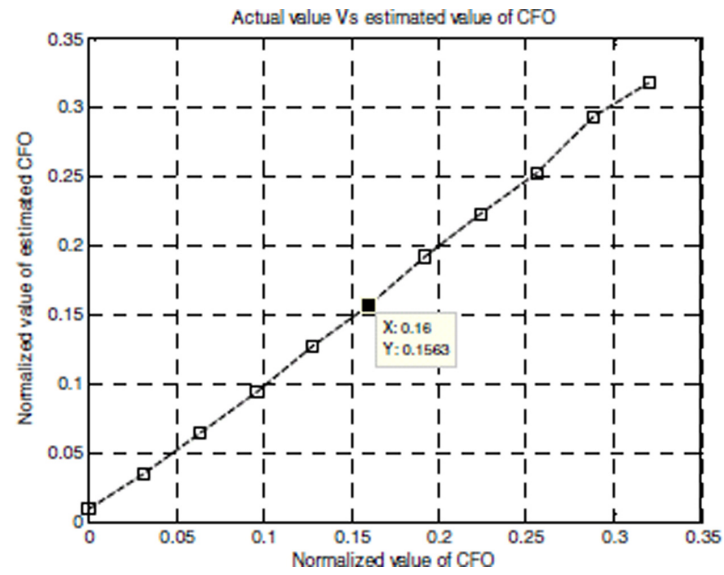

Figure 12 Actual and estimated value of CFO

of actual $\mathrm{CFO}$ versus estimated CFO. One data cursor in figure 12 is displaying both of these values. Values of $\mathrm{CFO}$ here are normalized with respect to sub carrier spacing.

\section{Discussion and Conclusion}

This paper examined and compared performance of proposed algorithm with existing $\mathrm{S}$ and $\mathrm{C}$ algorithm over AWGN and Rayleigh channel. This proposed timing synchronization algorithm is simple $\&$ the results are encouraging. Performance parameters like mean error, standard deviation of timing error estimation and detection probability are used. New algorithm shows superior 
performance to the existing $\mathrm{S}$ and $\mathrm{C}$ algorithm. Same training symbol and same function of timing estimation is used for CFO estimation. This reduces extra overhead and hence improves throughput of system.

\section{References}

[1] D. K. Kim, S. H. Do, H. B. Cho, H. J. Choi and K. B. Kim. A New Joint Algorithm Of Symbol Timing Recovery And Sampling Clock Adjustment For OFDM Systems, IEEE Transactions on Consumer Electronics, vol. 44, No.3, August 1998.

[2] J. J. van de Beek, M. Sandell, P. O. Borjesson. ML estimation of time and frequency offset in OFDM systems, Signal Processing, IEEE Transactions on [see also Acoustics, Speech, and Signal Processing, IEEE Transactions Volume 45, Issue 7, Page(s):1800-1805, July 1997.

[3] B. Ai, Member, IEEE, J.-h. Ge, Y. Wang, Member, IEEE, S. Y. Yang, and P. Liu. Decimal Frequency Offset Estimation in COFDM Wireless Communications, 154 IEEE Transactions on broadcasting, vol. 50, no. 2, June 2004.

[4] O. Ureten and Selc uk Tas, ciŏglu. Autocorrelation Properties Of OFDM Timing Synchronization Waveforms Employing Pilot Subcarriers, Research Article EURASIP Journal on Wireless Communications and Networking.

[5] D. Landstrijml, S. Kate, W. J.-J. van de Beek. Per Odling' Per O. Borjesson'. Symbol time offset estimation in coherent OFDM systems" 1999 IEEE.

[6] M. K. Morelli, C.-C. J. Pun, M.-O. Synchronization Techniques for Orthogonal Frequency Division Multiple Access (OFDMA): A Tutorial Review, Proceedings of the IEEE, Volume: 95, Issue: 7, On page(s): 1394-1427, July 2007.

[7] T. M. Schmidl and D. C. Cox. Robust frequency and timing synchronization for OFDM, IEEE Trans. on Commun., vol. 45, no. 12, pp. 1613-1621, Dec. 1997.

[8] K. Shi and B. E. Serpedin. Coarse frame and carrier synchronization of OFDM systems: A new metric and comparison", IEEE Trans. Wireless Commun., vol. 3, no. 4, pp. 1271-1284, Jul. 2004.

[9] J. Kim, J. Noh, K. H. Chang. An efficient timing synchronization method for OFDMA system, Wireless Communications and Applied Computational Electromagnetics, 2005. IEEE/ACES International Conference on 3-7 April 2005 Page(s):1018-1021. 
[10] H. Puska and H. Saarnisaari. Matched Filter Time and Frequency Synchronization Method for Ofdm Systems Using PN-Sequence Preambles, The 18th Annual IEEE International Symposium on Personal, Indoor and Mobile Radio Communications (PIMRC'07) University of Oulu, Centre for Wireless Communications (CWC).

[11] Channel Models : A Tutorial V1.0 by Raj Jain February 21, 2007.

\section{Biographies}

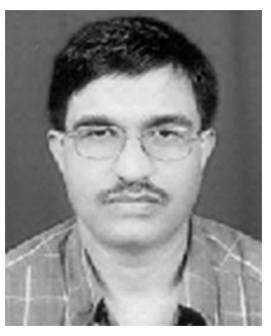

Abhay S. Gandhi did his BE (Electronic Engg) in 1989 from Visvesvaraya Regional College of Engineering (VRCE), Nagpur and ME (ECE) from Indian Institute of Science, Bangalore in 1991. After working for 3 years in industry and education, he joined VRCE (Now VNIT), Nagpur as Lecturer in July 1994. He has completed his PhD in August 2002. Currently, he is working as Professor at Department of Electronics Engineering, VNIT.

$\mathrm{He}$ has published 26 research papers in international conferences and journals. His research interests include signal processing, wireless digital communication, radio frequency (RF) circuits, and computer networks. E-mail: asgandhi@ece.vnit.ac.in

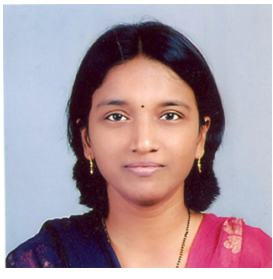

Anagha Rathkanthiwar did her BE (Electronic Engg) and M.Tech. (Electronics) in 1992 and 2003 respectively from RTMNU Nagpur (Nagpur University). She is pursuing her Ph.D. from Vesvesvaraya National Institute of Technology (VNIT), Nagpur. Currently, she is working as Assistant Professor 
in Electronics Engineering Department, Priyadarshini College of Engineering, Nagpur, Maharashtra, India.

She has published 14 research papers in international conferences and journals. Her research interests include signal processing, wireless communication. She has reviewed several research papers of International and National conferences. Also she has chaired sessions at National Conferences. E-mail: anagharathkanthiwar@yahoo.co.in 